\title{
Role of Complement-Derived and Bacterial Formylpeptide Chemotactic Factors in the In Vivo Migration of Neutrophils in Experimental Escherichia coli Pyelonephritis in Rats
}

\author{
Pascal R. Meylan and Michel P. Glauser
}

\author{
From the Division des Maladies Infectieuses of the \\ Département de Médecine Interne, Centre Hospitalier \\ Universitaire Vaudois, Lausanne, Switzerland
}

\begin{abstract}
In experimental Escherichia coli pyelonephritis, the bacterial multiplication in the kidney parenchyma triggers a burst of neutrophil extravascular migration, as measured by the myeloperoxidase (MPO) activity in the kidney, a marker for tissue neutrophil infiltration. To test the mechanisms of in vivo neutrophil migration, pyelonephritis was surgically induced in rats that were then either complement-depleted with cobra venom factor (CVF), resulting in a profound hypocomplementemia for $72 \mathrm{~h}$ after inoculation, or treated with phenylbutazone ( $\mathrm{PB})$, a competitive antagonist of bacterial chemotactic formylpeptides. Compared to controls, CVF- and PB-treated animals killed when the neutrophil infiltration started $(32 \mathrm{~h})$ had a significantly reduced neutrophil infiltration, as measured by kidney MPO activity. This effect disappeared in animals killed $72 \mathrm{~h}$ after surgery, when neutrophil infiltration peaked. These data suggest that redundant chemotactic mechanisms triggered neutrophil migration. Inhibiting one of these mechanisms only transiently delayed neutrophil migration but did not affect the peak infiltration.
\end{abstract}

Recent experiments using a rat model of ascending obstructive Escherichia coli pyelonephritis have delineated the course of neutrophil polymorphonuclear leukocyte infiltration in response to the multiplication of bacteria in the kidney parenchyma [1]. This model therefore offers the opportunity for studying in vivo the mechanisms of neutrophil migration. In vitro experiments using the filter assay or the "under-agarose migration assay" have demonstrated that gradients of various chemoattractants trigger chemotaxis, a directional migration of neutrophils [2]. The best known chemoattractants are the complement-derived C5a and C5a-des-arg peptides [3-5], the bacterial formylpeptides [6,7], and oxidized derivatives of arachidonic acid generated through the lipooxygenase pathway, mainly leukotriene B4 [8]. In vivo, the injection of these substances promotes the extravascular migration of neu-

Received for publication 11 July 1988 and in revised form 4 November 1988.

- This work was supported in part by grant 8.836 .81 from the Swiss National Foundation for Scientific Research.

The authors thank Marlies Knaup for technical and Jacqueline Mayor for secretarial assistance.

Please address requests for reprints to Dr. Michel P. Glauser, Division des Maladies Infectieuses, Département de Médecine Interne, Centre Hospitalier Universitaire Vaudois, 1011 Lausanne, Switzerland. trophils [9]. During acute $E$. coli pyelonephritis, bacteria multiplying within the kidney parenchyma may produce chemotactic formylpeptides [7] and activate the complement cascade by its alternate pathway [10].

We used two approaches to assess the respective role of these chemotactic substances in triggering the migration of neutrophils into the kidney interstitial space. First, cobra venom factor (CVF) was used to deplete circulating complement in rats. Hypocomplementemia was checked by measuring levels of total serum hemolytic complement $\left(\mathrm{CH}_{50}\right)$ and immunoreactive $\mathrm{C} 3$ and the capacity of the serum to generate in vitro neutrophil migration upon zymosan activation. Second, rats were treated with phenylbutazone (PB), a competitive inhibitor of chemotactic formylpeptides for neutrophil receptors [11-13], in doses that prevented formylpeptide-induced neutropenia in rabbits [12]. Some of the animals were killed during the acute phase of pyelonephritis, and the neutrophil infiltration of their kidney parenchyma was determined by measuring the myeloperoxidase (MPO) activity, an enzymatic marker for neutrophil infiltration [1]. Another group of animals was killed during the chronic phase of pyelonephritis, with the aim of correlating the effect of any diminished neutrophil migration during the acute phase with the development of pyelonephritic scarring. 


\section{Materials and Methods}

In vitro experiments. (1) Complement level determinations. The $\mathrm{CH}_{50}$ was measured according to established procedures [14]. Immunoreactive C3 levels were determined by rocket immunoelectrophoresis as described by Laurell [15] using a goat antiserum to rat C3c (Nordic Immuno, Tilburg, Netherlands).

The serum chemotactic activity upon zymosan activation was determined in an under-agarose migration assay. Pooled normal rat serum and test sera were activated as follows. Quantities ( $5 \mathrm{mg}$ each) of washed zymosan (Sigma Chemical, St. Louis) were added to $1-\mathrm{mL}$ aliquots of serum, which were vortexed and incubated $60 \mathrm{~min}$ at $37^{\circ} \mathrm{C}$. After centrifugation at $1000 \mathrm{~g}$ for $15 \mathrm{~min}$, the supernatant was harvested and stored at $-70^{\circ} \mathrm{C}$ until tested. Agarose plates $(0.75 \%)$ were prepared by heating $0.3 \mathrm{~g}$ of agarose (Seakem LE; FMC, Rockland, Me) in $32 \mathrm{~mL}$ of distilled water to $100^{\circ} \mathrm{C}$. After cooling to $50^{\circ} \mathrm{C}$, $4 \mathrm{~mL}$ of warm Medium 199 (concentration 10X) containing Earle's salts (GIBCO Laboratories, Paisley, UK), $4 \mathrm{~mL}$ of $7.5 \%$ bovine serum albumin, and $665 \mu \mathrm{L}$ of $7.5 \% \mathrm{NaHCO}_{3}$ was added. Aliquots ( $2 \mathrm{~mL}$ each) were poured into $35 \times 10-\mathrm{mm}$ Petri dishes (Falcon, Oxnard, Calif) and allowed to solidify. Four pairs of 2.4-mm-diameter wells were punched out $4.6 \mathrm{~mm}$ apart in each dish and the plugs gently removed by suctioning with a Pasteur pipette [16]. The attractant well was loaded with $5 \mu \mathrm{L}$ of zymosan-activated serum and left to prediffuse for $2 \mathrm{~h}$ at $37^{\circ} \mathrm{C}$ in a humidified incubator with $7 \% \mathrm{CO}_{2}$. Neutrophils were then loaded $(5 \mu \mathrm{L}$ containing $2 \times$ $10^{5}$ cells) into the opposite well and left to migrate for $2 \mathrm{~h}$ at $37^{\circ} \mathrm{C}$ with $7 \% \mathrm{CO}_{2}$. Migration was stopped by cooling at $4^{\circ} \mathrm{C}$ until the cells were counted. Cells migrating out of the well were counted using a Nikon inverted microscope with an ocular grid (Leitz Periplan, Wetzlar, GDR) as described [16]. Each experiment was done five times. The number of neutrophils migrating out of the wells was proportional to the reciprocal dilution of the pooled normal rat serum. This relationship was used to determine the titers of serum chemotactic activity of test sera. All complement levels in test sera were expressed as percent of the level in pooled normal rat serum.

(2) Serum bactericidal activity against E. coli. The bactericidal effect of rat serum against $E$. coli O6:K5:H1 used for inducing pyelonephritis was tested as suggested by Taylor [17]. The bacteria were grown to midexponential phase (about $10^{7} \mathrm{cfu} / \mathrm{mL}$ ) in Tryptone soy broth (GIBCO), harvested by centrifugation, and resuspended in gelatin-veronalcalcium-magnesium buffer (Bio-Mérieux, Lyon, France) containing various concentrations of fresh or heated $\left(56^{\circ} \mathrm{C}, 1 \mathrm{~h}\right)$ pooled normal rat serum to a final volume of $1 \mathrm{~mL}$. The test tubes were incubated in a rotating water bath at $37^{\circ} \mathrm{C}$. Aliquots were pipetted out after $0,1,2,3$, and $6 \mathrm{~h}$ of incubation and plated onto Columbia agar (GIBCO) with an automatic device (Spiral System DS; IG, Zurich). Colonies were counted after $24 \mathrm{~h}$ of incubation at $37^{\circ} \mathrm{C}$ and viable counts were expressed as $\log _{10} \mathrm{cfu} / \mathrm{mL}$.

In vivo experiments. (1) Experimental design. Ascending pyelonephritis was surgically induced in rats as previously described [18, 19]. After recovering from anesthesia, the rats were randomly assigned to one of three treatment groups. A first group received two iv injections of 100 units of CVF (Lot S 4131, Cordis Laboratories, Miami) 1 and $32 \mathrm{~h}$ after surgery. A control group received an identical schedule of iv saline. A third group received im phenylbutazone, $50 \mathrm{mg} / \mathrm{kg}$ per day for $3 \mathrm{~d}$ in two divided doses, the first dose being administered on the morning before surgery and the last dose at 72 $h$ after surgery. One-third of the animals in each treatment group were killed $32 \mathrm{~h}$ after surgery (the time when neutrophil migration started), $72 \mathrm{~h}$ after surgery (the time of peak neutrophil infiltration), or 2 mo after surgery (when chronic kidney scarring was fully established) [18]. Rats were killed as described [1].

In each experiment, parameters were evaluated as follows. The incidence of gross, macroscopic pyelonephritis was expressed as the number of rats with macroscopic lesions of the left kidney over the total number of rats. Kidneys that do not display pyelonephritic lesions harbor very low bacterial counts for a few days after inoculation and indicate a failure to induce pyelonephritis $[19,20]$. Bacterial counts in the kidney parenchyma were expressed as $\log ^{10}$ $\mathrm{cfu} / \mathrm{g}$ kidney tissue. In addition, the severity of the inflammatory changes was assessed by measuring the kidney weight, which provides a quantitative index of the severity of pyelonephritis. It increases in proportion to suppuration during acute pyelonephritis, whereas it decreases in proportion to the destruction of kidney tissue during chronic pyelonephritis $[1,18,19]$. To minimize the effects of kidney weight variation among animals, the ratio of the weight of the left to the weight of the right kidney (L/R kidney weight ratio) was used. This ratio also expresses 
the compensatory hypertrophy of the right kidney that takes place during chronic pyelonephritis in proportion to the extent of left kidney parenchyma destruction [18]. This has been confirmed by functional radioisotopic studies [1].

The kidney MPO activity in the kidney homogenates, which has been shown to be a specific and sensitive marker for kidney neutrophil infiltration, was also measured [1]. This activity was expressed as MPO activity units per kidney, taking into account both the kidney weight and the dilution due to homogenization. Blood samples from CVF-treated and control rats killed during the acute phase were left to clot for $1 \mathrm{~h}$ at room temperature. The serum was harvested and stored at $-70^{\circ} \mathrm{C}$ before complement level determinations.

(2) Preparation of isolated neutrophils. Isolated neutrophils for in vitro testing were obtained in rats by ip injection of $5 \mathrm{~mL}$ of $30 \mathrm{mg} / \mathrm{mL}$ Thioglycollate broth (Difco Laboratories, Detroit) containing $2 \mathrm{IU} / \mathrm{mL}$ of heparin (Heparin Kabi; Kabi $\mathrm{AB}$, Stockholm). The peritoneal cavity was opened aseptically $4 \mathrm{~h}$ later and washed with $10 \mathrm{~mL}$ of icecold Earle's balanced salt solution containing 7.5 $\mathrm{mg} / \mathrm{mL}$ of bovine serum albumin (GIBCO) and $2 \mathrm{IU} / \mathrm{mL}$ of heparin. The washing fluid was harvested and the peritoneal cells were centrifuged at $100 \mathrm{~g}$, for $10 \mathrm{~min}$. Hypotonic lysis of the red cells was performed in distilled water $(30 \mathrm{sec})$. Isotonicity was restored by concentrated Earle's. The cells were washed again by centrifugation, resuspended in Earle's, and counted in a hemocytometer. Each rat yielded $\sim 2 \times 10^{7}$ cells. Trypan blue exclusion indicated more than $95 \%$ viability. Differential counts on Wright's stained smears indicated that more than $98 \%$ of the cells were polymorphonuclear leukocytes.

(3) Statistical analysis. In vivo experiments were performed twice and, because they gave reproducible results, the data were pooled for statistical analysis. Linear correlations were computed by the leastsquares method. Comparisons between groups were done by the two-tailed Wilcoxon rank sum test. To take into account the fact that three groups were compared, the Bonferroni adjustment was used [21] and the level of significance set at $P=.025$.

\section{Results}

In vitro experiments. The under-agarose migration assay was used as a functional assay for serum factors able to produce chemotactic substances upon zymosan activation, especially serum C5 $[3,5]$. Indeed, the number of cells migrating toward the attracting well was proportional to the concentration of zymosan-activated pooled normal rat serum (figure 1), suggesting that cell migration is an indicator of the chemotactic substances in activated serum. Figure 2 demonstrates the effect of CVF treatment in rats with pyelonephritis on the $\mathrm{CH}_{50}$, immunoreactive $\mathrm{C} 3$, and functional $\mathrm{C} 5$ levels. In rats killed 32 $\mathrm{h}$ after surgery, the $\mathrm{CH}_{50}, \mathrm{C} 3$, and functional $\mathrm{C} 5$ levels were profoundly depressed in CVF-treated rats compared with those in controls. In the group of rats killed $72 \mathrm{~h}$ after surgery, $\mathrm{CH}_{30}$ levels in CVF-treated rats increased slightly but remained profoundly depressed while $\mathrm{C} 3$ and functional $\mathrm{C} 5$ levels were even lower than those in animals killed $32 \mathrm{~h}$ after surgery. Preliminary experiments (data not shown) have demonstrated that similar doses of CVF profoundly depressed the complement levels within a few hours after the injection. Thus, CVF-treated rats had very low complement levels from the time of sur-

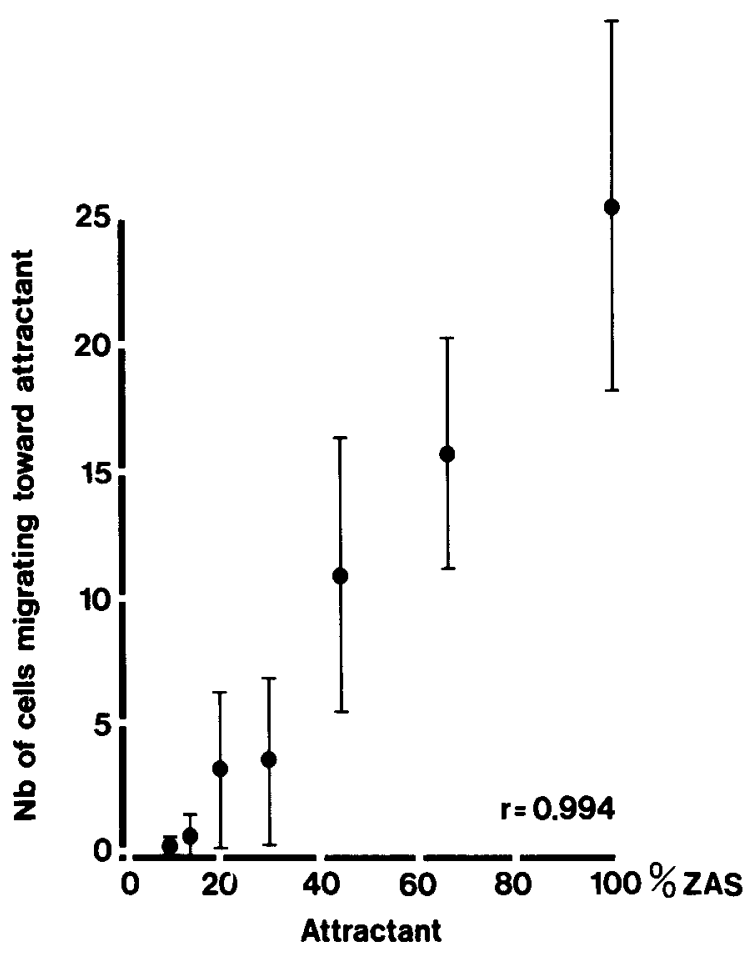

Figure 1. Effect of zymosan activated serum (ZAS) on neutrophil migration in the agarose assay, as measured by the number of neutrophils migrating toward the attracting well. Each point represents the mean and standard deviation of five assays. 


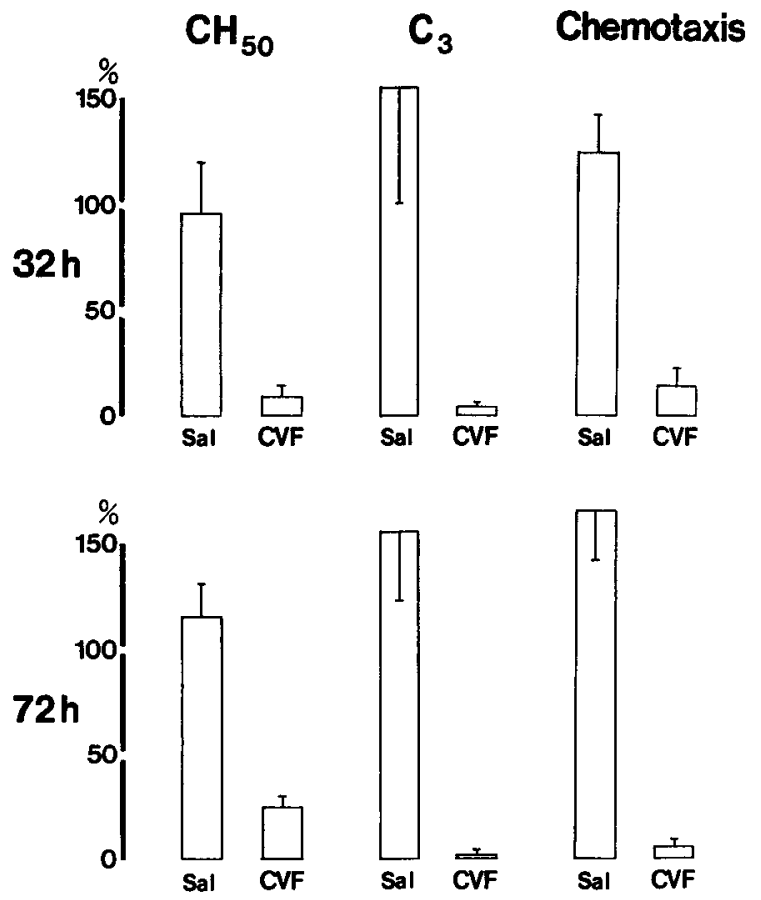

Figure 2. Complement levels in animals with pyelonephritis killed 32 and $72 \mathrm{~h}$ after the inoculation. $\mathrm{CH}_{\mathbf{5 0}}$ : total serum hemolytic complement; C3: immunoreactive C3; Chemotaxis: serum chemotactic activity upon zymosan activation, i.e., functional assay for serum C5. Sal = saline-treated (controls); CVF = CVF-treated animals. Results are expressed as percent of the level found in pooled normal rat serum. Columns represent means, and bars indicate standard deviations of each group. gery until $72 \mathrm{~h}$ afterwards, and perhaps for a longer time. In contrast, saline-treated animals with pyelonephritis had higher complement levels than those found in pooled normal rat serum, probably resulting from the acute inflammatory reaction.

In vivo experiments. Overall, 176 rats had surgery to produce pyelonephritis. Fourteen of them died soon after surgery, because of the anesthesia or $E$. coli septicemia secondary to severe pyelonephritis as suggested by post-mortem examination and cultures. As shown in table 1, these early deaths occurred mainly among the CVF-treated animals and to a lesser degree among PB-treated animals killed $72 \mathrm{~h}$ or $2 \mathrm{mo}$ after surgery. Therefore, in these treatment groups, the disappearance of those animals with the most severe pyelonephritis before the planned time of sacrifice might have introduced a bias toward underestimating the severity of pyelonephritis. In addition, neither CVF nor PB treatment significantly influenced the incidence of pyelonephritis (table 1).

In animals killed $32 \mathrm{~h}$ after surgery, both CVFand PB-treatment significantly reduced MPO activity in their left kidneys compared to that in controls (figure 3). The median MPO activity was respectively 19.6 activity units per kidney (CVF) and 13.1 activity units per kidney (PB) compared to 53.1 activity units per kidney in controls $(P<.01$ for both comparisons). Thus, both treatments significantly reduced the early neutrophil migration into the kid-

Table 1. Effect of cobra venom factor (CVF) and phenylbutazone (PB) treatment on pyelonephritis.

\begin{tabular}{|c|c|c|c|c|c|c|c|}
\hline \multirow{4}{*}{$\begin{array}{l}\text { Time of } \\
\text { sacrifice*; treatment }\end{array}$} & \multirow{2}{*}{\multicolumn{3}{|c|}{ No. of animals }} & \multicolumn{4}{|c|}{ Severity of pyelonephritis } \\
\hline & & & & & & & \\
\hline & \multirow[b]{2}{*}{ Operated } & \multirow{2}{*}{$\begin{array}{c}\text { Surviving } \\
\text { until } \\
\text { sacrifice }\end{array}$} & \multirow{2}{*}{$\begin{array}{l}\text { With pyelo- } \\
\text { nephritis at } \\
\text { sacrifice }(\%)\end{array}$} & \multicolumn{2}{|c|}{$\begin{array}{c}\text { Overall inflammatory } \\
\text { response }\end{array}$} & \multicolumn{2}{|c|}{ Bacterial counts $\ddagger$} \\
\hline & & & & median (range) & $P$ & median (range) & $P$ \\
\hline \multicolumn{8}{|l|}{$32 \mathrm{~h}$} \\
\hline Saline & 20 & 20 & $16(80)$ & $1.39(1.21-1.54)$ & & $8.36(7.81-9.09)$ & \\
\hline CVF & 19 & 19 & $15(79)$ & $1.36(1.11-1.57)$ & .79 & $8.18(7.39-9.06)$ & .15 \\
\hline PB & 20 & 20 & $16(80)$ & $1.24(1.13-1.48)$ & $<.01$ & $7.92(6.41-8.45)$ & $<.01$ \\
\hline \multicolumn{8}{|l|}{$72 \mathrm{~h}$} \\
\hline Saline & 18 & 17 & $11(65)$ & $1.76(1.09-2.41)$ & & $8.84(6.50-10.17)$ & \\
\hline CVF & 20 & 20 & $18(90)$ & $2.00(1.42-2.55)$ & .15 & $9.43(8.79-10.24)$ & .035 \\
\hline PB & 20 & 17 & $15(83)$ & $1.52(0.92-1.77)$ & .04 & $8.67(7.76-9.74)$ & .31 \\
\hline \multicolumn{8}{|l|}{$2 \mathrm{mo}$} \\
\hline Saline & 20 & 20 & $18(90)$ & $0.65(0.10-0.88)$ & & $4.93(1.81-8.08)$ & \\
\hline CVF & 20 & 13 & $13(100)$ & $0.64(0.22-0.97)$ & .94 & $5.10(1.75-7.05)$ & .61 \\
\hline PB & 19 & 16 & $14(88)$ & $0.47(0.17-0.98)$ & .52 & $4.50(1.73-6.78)$ & .59 \\
\hline
\end{tabular}

NOTE. $P$ values refer to statistical comparisons (Wilcoxon) of each treatment group with controls.

* After surgery.

Ratio of the weight of the left to the weight of the right kidney from animals with pyelonephritis.

$\ddagger \log _{10} \mathrm{cfu} / \mathrm{g}$ kidney tissue of animals with pyelonephritis. 


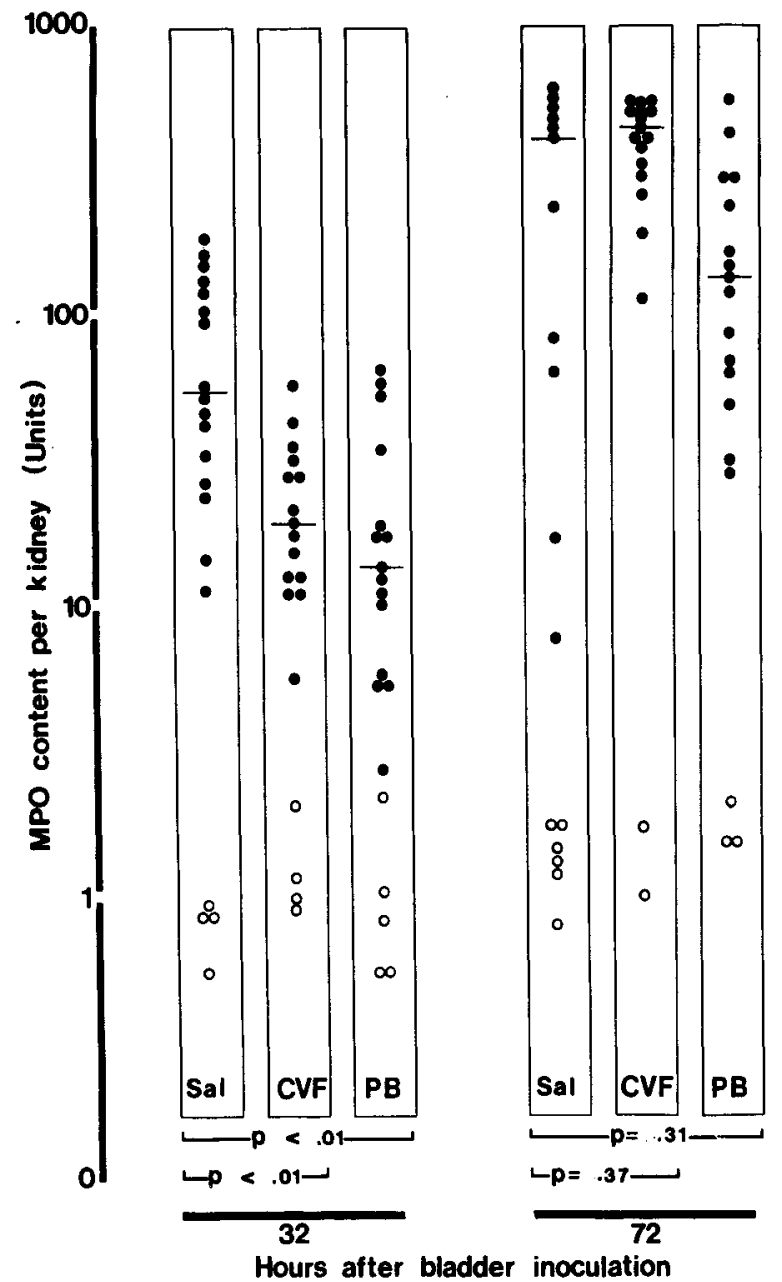

Figure 3. Left kidney myeloperoxidase (MPO) activity of animals sacrificed 32 or $72 \mathrm{~h}$ after inoculation. Sal: saline-treated (controls); CVF: cobra venom factor-treated; PB: phenylbutazone-treated. Closed circles represent the kidney MPO activity of animals with macroscopic signs of pyelonephritis and high bacterial counts, while open circles are from animals without macroscopic pyelonephritis and with very low bacterial counts $\left(<1 \mathrm{O}_{2} \mathrm{cfu} / \mathrm{g}\right.$ of kidney tissue). Bars represent medians. $P$ values (two-tailed Wilcoxon) are indicated beneath the columns.

ney parenchyma. However, despite continued CVF or PB therapy until $72 \mathrm{~h}$ after inoculation, there was no longer any significant reduction in the left kidney MPO activity in CVF- or PB-treated animals ( $P=.37$ and .31 , respectively). These findings show that the initially reduced neutrophil infiltration was transient, probably related to a delayed migration induced by each treatment.

PB diminished the acute inflammatory swelling of kidneys, as measured by the L/R kidney weight ratio (table 1). This antiinflammatory effect of PB was accompanied by a transient reduction of the bacterial counts in the pyelonephritic kidneys early after the production of pyelonephritis $(32 \mathrm{~h})$. In contrast, CVF treatment not only did not prevent the inflammatory swelling, but also enhanced bacterial multiplication to a near significant degree in the kidney of animals killed $72 \mathrm{~h}$ after surgery, a fact probably related to the serum susceptibility of the $E$. coli study strain (data not shown). When the rats were killed 2 mo after surgery, the $L / R$ kidney weight ratio was similar in CVF-, PB-, and saline-treated animals, reflecting a degree of pyelonephritic scarring of similar severity.

\section{Discussion}

The present experiments demonstrated that both CVF and PB treatments were able to transiently reduce the in vivo neutrophil migration early during pyelonephritis, an effect that was no longer apparent at the time of peak kidney neutrophil infiltration [1]. It is possible that this transient effect of both treatments on neutrophil migration might have been a result of the CVF or PB treatment becoming ineffective beyond $32 \mathrm{~h}$ after surgery.

With regard to CVF treatment, this was probably not the case, because the CVF produced a prolonged hypocomplementemia that lasted throughout the treatment period. Particularly, the ability of serum to produce chemotactic factors upon zymosan activation was strikingly depressed in CVF-treated rats as demonstrated using the under-agarose migration assay. In addition, in studies using animals used in the present experiments and fluorescent antibodies to rat C3, no CVF-treated animal shed demonstrable C3-coated $E$. coli in the urine during acute pyelonephritis, in contrast to brisk fluorescent bacilli observed in the urine of a majority of control animals. In vitro experiments using fresh pooled normal rat serum to coat $E$. coli in urine demonstrated that fluorescence was observed with serum dilutions as high as 1:200 [22]. These observations suggest, therefore, that complement levels within the kidney tissue were also effectively depressed by CVF treatment.

PB treatment was administered in the present study at doses that others have shown to completely prevent the neutropenia induced in rabbits by iv injection of the formylpeptide formyl-methionylleucyl-phenylalanine [12]. Although a different efficacy of this drug in the rat can not be excluded, 
its effect on early neutrophil migration suggests that the present doses were effective. We hypothesize that PB inhibited early neutrophil infiltration by interfering with bacterial formylpeptide-mediated chemotaxis. However, it is possible that $\mathrm{PB}$ acted by another mechanism involving inhibition of prostaglandin synthesis.

In various experimental models of $E$. coli pyelonephritis, conflicting results have been reported with respect to the effect of decomplementation on neutrophil infiltration and inflammatory damage. Sullivan et al. [23], using a rat model of hematogenous pyelonephritis, as well as Roberts et al. [24], using a model of ascending pyelonephritis in rhesus monkeys, demonstrated a reduced neutrophilic infiltration in CVF-treated animals early after infection, but observations over periods longer than 48 $h$ were not performed. In contrast, Wilson et al. [25], using a rat model of pyelonephritis induced by direct intrarenal inoculation, did not observe a reduced neutrophil infiltration in CVF-treated animals followed for $4 \mathrm{~d}$. Thus, these contradictory results might reflect the fact that the short-term studies detected transient reduction of neutrophil infiltration that was not shown by studies with longer periods of observation.

In experimental models of bacterial diseases other than pyelonephritis, similar findings of delayed neutrophil exudation have been observed. For instance, Tuomanen et al. [26], using rabbits with experimental Streptococcus pneumoniae meningitis treated with CVF, observed a delayed rise in cerebrospinal fluid leukocyte counts. Toews et al. $[27,28]$ observed in experimental pneumococcal and Hemophilus influenzae pneumonia a significant neutrophil recruitment into the lung of congenitally C5-deficient mice. They concluded that chemotactic factors other than C5a were produced in the alveoli, demonstrating the redundancy of the chemotactic mechanisms after pulmonary challenge with $S$. pneumoniae or $H$. influenzae. Similar conclusions might be drawn from the results of the present experiments. Indeed, the lack of a sustained efficacy of both CVF and PB treatment at reducing the peak neutrophil infiltration observed here suggests that several independent chemotactic mechanisms operated during acute pyelonephritis. We postulate that complement-derived as well as bacterial formylpeptide chemotactic factors played an important role in triggering the neutrophil migration during the early inflammatory response in vivo. Later on, other concurrent chemo- tactic mechanisms became powerful enough to induce a neutrophil migration despite continued complement depletion or inhibition of formylpeptide receptors.

Recent evidence suggests that endothelial recognition determinants for neutrophil adherence molecules may play a role in regulating the extravascular migration of inflammatory cells into inflamed tissue [29]. These determinants are upregulated by lipopolysaccharide and other inflammatory mediators and can facilitate the attachment of neutrophils to the endothelium even in the absence of chemotactic factors. They can also act with chemotactic factors to enhance transendothelial migration. Therefore, in addition to chemotactic factors acting on inflammatory cells, neutrophil infiltration might be partially regulated by inflammatory substances acting on endothelial cells. These observations might thus be a concurrent explanation for neutrophil infiltration apparently independent of chemotactic factors.

In the rat model of ascending pyelonephritis, chronic renal scarring follows the irreversible tissue damage that results from acute inflammatory processes $[1,18,19]$. In the present experiments, neither CVF nor PB treatment resulted in any significant protection against permanent kidney damage. This observation is not invalidated by the early deaths of CVF-treated animals to be killed at $72 \mathrm{~h}$ and 2 mo after surgery. Indeed, the disappearance of those rats with the most severe pyelonephritis might only lead to an underestimation of the median severity of pyelonephritis. This supports the interpretation that pyelonephritis was at least as severe in CVFtreated animals as in controls at these later times of sacrifice. The fact that higher bacterial counts were observed during acute pyelonephritis in CVF-treated animals supports our previous findings $[1,19,30]$ that excessive infiltration and activation of neutrophils during the acute phase of pyelonephritis, not bacterial virulence factors, is the major culprit responsible for the tissue damage that leads to pyelonephritic scarring.

In conclusion, both CVF and $\mathrm{PB}$ treatments reduced early neutrophil infiltration during acute pyelonephritis. However, this reduction was only transient; peak neutrophil infiltration was not reduced. This suggests that complement-derived as well as bacterial formylpeptide chemotactic factors played an important role in triggering neutrophil migration during the early inflammatory response. Later on, 
other concurrent chemotactic mechanisms became powerful enough to induce a neutrophil migration despite continued complement depletion or inhibition of formylpeptide receptors. The similar peak neutrophil infiltration resulted in chronic kidney scarring of similar severity among the various treatment groups, supporting the hypothesis that neutrophils, rather than bacterial virulence factors, play the major role in the pathogenesis of pyelonephritic scarring.

\section{References}

1. Meylan PR, Glauser MP. Failure of dexamethasone to prevent polymorphonuclear leukocyte infiltration during experimental acute exudative pyelonephritis and to reduce subsequent chronic scarring. J Infec Dis 1988;157:480-485

2. Schiffmann E. Leukocyte chemotaxis. Ann Rev Physiol 1982;44:553-568

3. Goldstein IM. Complement: biologically active products. In: Gallin JI, Golstein IM, Snyderman R, eds. Inflammation: basic principles and clinical correlates. New York: Raven Press, 1988:55-74

4. Ward PA, Cochrane CG, Müller-Eberhard HJ. The role of serum complement in chemotaxis of leukocytes in vitro. J Exp Med 1965;122:327-346

5. Fernandez HN, Hugli TE. Primary structural analysis of the polypeptide portion of human C5a anaphylatoxin. J Biol Chem 1978;253:6955-6964

6. Schiffmann E, Showell HV, Corcoran BA, Ward PA, Smith $E$, Becker EL. The isolation and partial characterization of neutrophil chemotactic factors from Escherichia coli. J Immunol 1975;114:1831-1837

7. Marasco WA, Phan SH, Krutzsch H, Showell HJ, Feltner DE, Narin R, Becker EL, Ward PA. Purification and identification of formyl-methionyl-leucyl-phenylalanine as the major peptide neutrophil chemotactic factor produced by Escherichia coli. J Biol Chem 1978;259:5430-5439

8. Palmblad J, Malmsten CL, Udén AM, Rådmark $\mathrm{O}$, Engstedt L, Samuelsson B. Leukotriene B4 is a potent and stereospecific stimulator of neutrophil chemotaxis and adherence. Blood 1981;58:658-661

9. Movat HZ, Rettl C, Burrowes CE, Johnston MG. The in vivo effect of leukotriene B4 on polymorphonuclear leukocytes and the microcirculation. Am J Pathol 1984;115:233-244

10. Morrison DC, Kline LF. Activation of the classical and properdin pathways of complement by bacterial lipopolysaccharides (LPS). J Immunol 1977;118:362-368

11. Nelson RD, Gracyk JM, Fiegel VD, Herron MJ, Chenoweth DE. Chemotactic deactivation of human neutrophils: protective influence of phenylbutazone. Blood 1981;58:752-758

12. Dahinden C, Fehr J. Receptor-directed inhibition of chemotactic factor-induced neutrophil hyperactivity by pyrazolone derivative. J Clin Invest 1980;66:884-891

13. Perianin A, Labro MT, Hakim J. Chemokinetic activity of $\mathrm{N}$-formyl-methionyl-leucyl-phenylalanine on human neutrophils and its modulation by phenylbutazone. Biochem Pharmacol 1982;31:3071-3076
14. Ruddy S. Complement. In: Rose NR, Friedman H, Fahay $\mathrm{JL}$, eds. Manual of clinical and laboratory immunology. 3rd ed. Washington, DC: American Society for Microbiology, 1986:175-184

15. Laurell CB. Electroimmunoassay. Scand J Clin Lab Invest [Suppl] 1972;124:21-23

16. Orr W, Ward PA. Quantitation of leukotaxis in agarose by three different methods. J Immunol Methods 1978;20: 95-107

17. Taylor PW. Bactericidal and bacteriolytic activity of serum against gram-negative bacteria. Microbiol Rev 1983;47: 46-83

18. Brooks SJD, Lyons JM, Braude AI. Immunization against retrograde pyelonephritis. III. Vaccination against chronic pyelonephritis due to Escherichia coli. J Infect Dis 1977;136:633-639

19. Glauser MP, Lyons JM, Braude AI. Prevention of chronic experimental pyelonephritis by suppression of acute suppuration. J Clin Invest 1978;61:403-407

20. Glauser MP, Francioli PB, Bille J, Bonard M, Meylan PR. Effect of indomethacin on the incidence of experimental Escherichia coli pyelonephritis. Infect Immun 1983;40: 529-533

21. Godfrey K. Comparing the means of several groups. N Engl J Med 1985;313:1450-1456

22. Paquet $\mathrm{N}$. Detection of complement-coated bacteria in experimental urinary tract infections. Master's thesis, Geneva: University of Geneva, 1984

23. Sullivan MJ, Harvey RA, Shimamura T. The effects of cobra venom factor, an inhibitor of the complement system, on the sequence of morphological events in the rat kidney in experimental pyleonephritis. Yale J Biol Med 1977; 50:267-273

24. Roberts JA, Roth JK Jr, Domingue G, Lewis RW, Kaack B, Baskin G. Immunology of pyelonephritis in the primate model. VI. Effect of complement depletion. J Urol 1983; 129:193-196

25. Wilson DM, Ormrod DJ, Miller TE. Role of complement in chemotaxis: study of a localized infection. Infect Immun 1980;29:8-12

26. Tuomanen E, Hengstler B, Zak O, Tomasz A. The role of the complement and arachidonic acid pathways in inflammation during pneumococcal meningitis [abstract 682]. In: Program and abstracts of the 25th Interscience Conference on Antimicrobial Agents and Chemotherapy. Washington, DC: American Society for Microbiology, 1985

27. Toews GB, Vial WC. The role of C5 in polymorphonuclear leukocyte recruitment in response to Streptococcus pneumoniae. Am Rev Respir Dis 1984;129:82-86

28. Toews GB, Vial WC, Hansen EJ. Role of C5 and recruited neutrophils in early clearance on nontypable Haemophilus influenzae from murine lungs. Infect Immun 1985; 50:207-212

29. Pohlman TH, Stanness KA, Beatty PG, Ochs HD, Harlan JM. An endothelial cell surface factor(s) induced in vitro by lipopolysaccharide, interleukin I, and tumor necrosis factor-alpha increases neutrophil adherence by a CDw18dependent mechanism. J Immunol 1986;136:4548-4553

30. Bille J, Glauser MP. Protection against chronic pyelonephritis in rats by suppression of acute suppuration: effect of colchicine and neutropenia. J Infect Dis 1982;146:220-226 